\title{
Instrumentos de Vigilância e Rastreio do Desenvolvimento Infantil e Tecnologia Móvel: Revisão
}

\author{
Flavia Monteiro Pereira ${ }^{1} \mathbb{D}$, Helenice Charchat-Fichman (D), J. Landeira-Fernandez (D) \\ Pontifícia Universidade Católica do Rio de Janeiro, Rio de Janeiro-RJ, Brasil
}

\section{RESUMO}

Este estudo é uma revisão de literatura desenvolvida para investigar o uso de instrumentos de vigilância e rastreio para atraso do desenvolvimento infantil por meio de tecnologia móvel. Além disso, objetivou-se caracterizar as metodologias utilizadas, amostras de participantes, se clínicas ou não, contexto de pesquisa, bem como a finalidade do uso instrumento. Foi realizada a busca de artigos, por três juízes, nas bases de dados indexadas na BVS, PUBMED/MEDLINE e APA PsyNET. Utilizaram-se as recomendações do PRISMA para essa revisão. A partir de critérios de inclusão/exclusão, foram recuperados e analisados cinco artigos. As duas modalidades de instrumentos: vigilância e rastreio, apresentaram-se em três categorias de estudo: 1 . descritivo; 2. viabilidade/usabilidade e 3. validade. Conclui-se que mais estudos sejam necessários, especialmente para avaliar o impacto e eficácia do uso de instrumentos de vigilância e rastreio para atraso no desenvolvimento infantil em formato de tecnologia móvel.

Palavras-chave: desenvolvimento infantil; rastreio; vigilância; $m$-Health.

\section{ABSTRACT - Child Development Monitoring and Screening Instruments and Mobile Technology: Review}

This study is a literature review designed to investigate the use of monitoring and screening instruments for child developmental delay using mobile technology. In addition, the aim was to characterize the methodologies used, samples of participants, whether clinical or not, the research context and the purpose of the instrument use. Articles were searched by three judges in the databases indexed in the BVS, PUBMED/MEDLINE and APA PsyNET. The PRISMA recommendations were used for this review. After applying the inclusion/exclusion criteria, five articles were retrieved and analyzed. The two types of instruments: monitoring and screening, were presented in three study categories: 1 . descriptive; 2. viability/usability and 3. validity. Further studies are needed to assess the impact and effectiveness of the use of mobile format instruments for the monitoring and screening for childhood developmental delays.

Keywords: child development; screening; monitoring; $m$-Health .

\section{RESUMEN - Instrumentos de Vigilancia y Detección del Desarrollo Infantil y Tecnología Móvil: Revisión}

Este estudio es una revisión de la literatura desarrollada para investigar el uso de instrumentos de vigilancia y rastreo del retraso del desarrollo infantil a través de la tecnología móvil. Asimismo, el objetivo fue caracterizar las metodologías utilizadas, muestras de participantes, ya sean clínicas o no; además del contexto de investigación y la finalidad del uso del instrumento. Tres jueces realizaron una búsqueda de artículos en las bases de datos indexadas en la BVS, PUBMED/MEDLINE y APA PsyNET. Las recomendaciones de PRISMA se utilizaron para esta revisión. Según los criterios de inclusión/exclusión, cinco artículos fueron recuperados y analizados. Los dos tipos de instrumentos fueron: vigilancia y detección, se presentaron en tres categorías de estudio: 1. descriptivo; 2. viabilidad/ usabilidad y 3. validez. Se concluyó que son necesarios más estudios, especialmente para evaluar el impacto y la efectividad del uso de instrumentos de vigilancia y rastreo del retraso en el desarrollo infantil en formato de tecnología móvil.

Palabras clave: desarrollo infantil; rastreo; vigilancia; $m$-Health .

A participação do acesso à internet por dispositivos móveis no Brasil dobrou entre o período de 2014 a 2017 (Centro Regional de Estudos para o Desenvolvimento da Sociedade da Informação [CETIC], 2017). Dados do Comitê Gestor da Internet no Brasil (CGI, 2018) revelam que o número de usuários de internet no país chegou a 120,7 milhões. Dessa população, quase a totalidade $(96 \%)$ usou a internet pelo telefone celular, sendo que $49 \%$ deles utilizaram a rede apenas por meio desse dispositivo.
Atualmente, a incorporação de novas tecnologias na prática profissional de psicólogos já é responsável por promover novas demandas e contextos de trabalho (Becker et al., 2018; Conselho Federal de Psicologia [CFP], 2018). No Brasil, o Conselho Federal de Psicologia representa o órgão regulador dessas práticas (CFP, 2018).

No entanto, as instituições regulatórias referentes ao acesso às práticas para utilização de instrumentos de avaliação, seja em formato "papel e lápis" ou computadorizados variam muito de país para país. Considerando-se

${ }^{1}$ Endereço para correspondência: Rua Marquês de São Vicente, 225, Gávea, 22451-900, Rio de Janeiro, RJ. E-mail: flaviamonteiropereira@hotmail.com Artigo derivado da Dissertação de Mestrado de Flávia Monteiro Pereira com orientação de J.Landeira-Fernandez, defendida em 2019 no programa de pós-graduação em Psicologia Clínica da Pontifícia Universidade Católica - Rio. 
esse fato, a International Guide Commission - ITC fornece em seu documento (International Test Commission [ITC], 2005) diretrizes internacionalmente aceitas sobre padrões éticos no uso de testes, competência e habilidade na aplicação, padrões de acesso, respeito aos direitos autorais e editoriais, assim como questões de segurança de instrumentos disponibilizados na internet.

Partindo-se dos pilares dessas diretrizes, os benefícios obtidos pela tecnologia garantem ao usuário soluções e inovações em atividades de pesquisa, clínica, avaliação psicológica, intervenção, reabilitação, aplicação de escalas, inventários de preenchimento on-line (Charchat-Fichman et al., 2014, Johnson \& Blasco, 1997; Primi, 2010; Twomey et al., 2018). As inovações em eletronic health (e-health) e mobile health (m-health) refletem a ascendência de pesquisas em andamento na interseção entre tecnologia e psicologia da saúde (Borrelli e Ritterband, 2015).

De acordo com a Associação Americana de Neurologia (AAN, Shevell et al., 2003), estima-se que de 1 a $3 \%$ das crianças com idade inferior a 5 anos são afetadas por déficit global no desenvolvimento. Quanto aos países subdesenvolvidos, os baixos registros e documentação impactam no acesso à dados estáticos epidemiológicos confiáveis referentes à prevalência e incidência de transtornos ou alterações no desenvolvimento (Durkin, 2002). Admite-se baixa prioridade da temática diante de taxas de mortalidade excessivas, pobreza e desnutrição (Grantham-Mcgregor et al., 2007).

$\mathrm{O}$ uso das nomenclaturas "atraso no desenvolvimento", "desenvolvimento desordenado" e "anormalidade de desenvolvimento" adotados como sinônimos, referem-se ao atraso no desenvolvimento normativo, onde os marcos existentes fornecem um parâmetro para observar e monitorar a criança ao longo do tempo (Duby et al., 2006). O termo é usualmente reservado para crianças abaixo de 5 anos de idade (Petersen et al, 1998)

Recentemente, a Academia Americana de Neurologia e o Comitê de Neurologia Infantil (Shevell et al., 2003) definem o atraso global no desenvolvimento como um subconjunto de deficiências no desenvolvimento e o atraso significativo é considerado quando ocorre discrepância de $25 \%$ ou mais da taxa esperada, ou uma diferença de 1,5-2,0 desvios padrão da norma em um ou mais domínios do desenvolvimento em testes norma-referenciados. Espera-se que sejam avaliados cinco domínios funcionais: comunicação, cognitivo, físico (motor fino/ grosso, audição,visão), social-emocional e adaptativo (Petersen et al., 1998; Shevell et al., 2003).

Destarte, seja na condição de desenvolvimento típico ou atípico, o incentivo ao uso de instrumentos de rastreio e/ou vigilância seja no formato "papel lápis" ou tecnológico, associa-se à potencialização do alcance de políticas públicas em saúde para identificação precoce (Duby et al., 2006; Gadomski et al., 2018, McLean et al., 2014). Etapa vital para o planejamento da intervenção precoce (Shonkoff \& Meisels, 2000; Hagan, Shaw, \& Duncan, 2017).

A vigilância (acompanhamento) do desenvolvimento é considerada um método de identificação precoce muito útil que pode ser realizado por uma ampla variedade de prestadores de serviços (Organização PanAmericana da Saúde [OPAS], 2005). A medida de vigilância inclui desde ouvir as preocupações familiares, mantendo um histórico preciso e registro até o uso de observações (Duby et al., 2006).

Embora seja um método valioso, a vigilância sozinha não é considerada suficientemente eficaz na detecção de atraso no desenvolvimento, pois está associada com pouca sensibilidade (Duby et al., 2006). Portanto, o emparelhamento das ferramentas de vigilância com o rastreio (triagem) de desenvolvimento é recomendado (Pizur-Barnekow et al., 2010).

Para que o rastreio do desenvolvimento seja eficaz, deve começar cedo na vida de uma criança e ser repetido ao longo da primeira infância (Duby et al., 2006). Recomenda-se usar instrumentos de rastreio confiáveis e válidos, apropriados às idades, cultura e linguagem da criança (Duby et al., 2006; Moodie et al., 2014). O que pode apresentar-se como um desafio, considerando-se o fato de que poucas ferramentas de rastreio de desenvolvimento são desenvolvidas ou testadas com amostras de crianças em outras línguas ou culturas (Moodie et al., 2014).

Visto que não existe uma ferramenta de rastreio universalmente aceita para todas as populações e todas as idades, esses procedimentos podem ser caracterizados como triagem amplas (multidimensional) ou restrito à domínios específicos, direcionados à uma área de suspeita diagnóstica (Duby et al., 2006; Halle, Zaslow, Wessel, Moodie e Darling-Churchill, 2011). Podendo apresentar-se na modalidade de preenchimento manual e/ou eletrônico (Moodie et al., 2014).

Nesse sentido, a incorporação desses recursos mediados pela tecnologia no âmbito das rotinas de atendimento em saúde e acompanhamento na educação já é uma realidade presente em alguns países (Agency for Health Care Research and Quality, 2015; Centre of Control Disease, U.S. Department of Health and Human Services Administration for Children and Families, 2015; CDC, 2017). No Brasil e internacionalmente, configura-se como uma área ainda em expansão e com potenciais desdobramentos (Primi, 2010; Koich Miguel, 2017; Borrelli \& Ritterband, 2015). O objetivo desta revisão é investigar quais foram os artigos que inseriram na metodologia do estudo a implementação de instrumento de vigilância ou rastreio para atraso de desenvolvimento por meio de aplicativo para tecnologia móvel ( $m$-health). E assim fomentar as melhores evidências para projeto de construção de um instrumento de rastreio em formato de aplicativo móvel para detecção de atraso do desenvolvimento infantil destinado à população brasileira. 


\section{Método}

O presente estudo inspirou-se nas recomendações da Preferred Reporting Items for Systematic Reviews and Meta-Analyses (PRISMA), que visam orientar a elaboração de revisões sistemáticas da literatura e metanálises na área da saúde humana (Moher et al., 2009) para explicar a busca e a seleção dos estudos, conforme fluxograma a seguir (Figura 1). O primeiro passo foi formular a questão da pesquisa que consistiu em "qual instrumento de vigilância ou rastreio para atraso de desenvolvimento para crianças de zero à cinco anos foi implementado em pesquisa por meio da tecnologia móvel?".

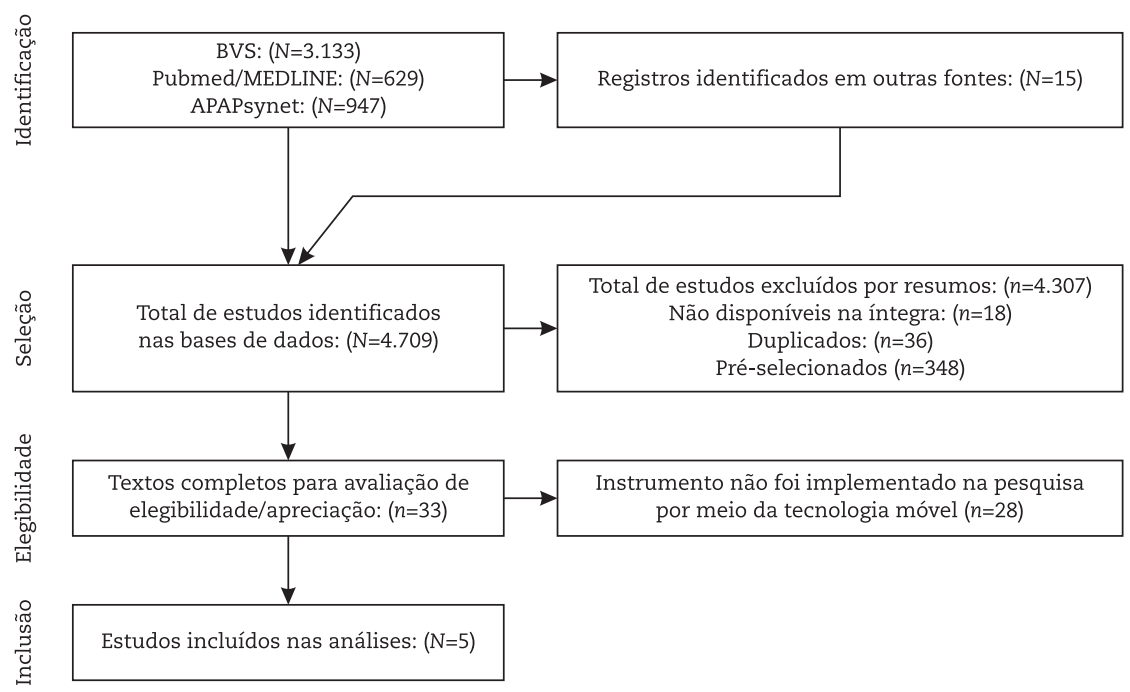

Figura 1. Fluxograma de busca e seleção dos artigos

A revisão da literatura será direcionada a responder à questão anterior. Será realizada busca a partir de descritores referentes ao tema do estudo, em português e inglês: Serão utilizadas três bases de dados nesse estudo: Biblioteca Virtual em Saúde - BVS, PUBMED/ MEDLINE e APA PsyNET. Foram selecionados os descritores conforme terminologia DeCS, da Biblioteca Virtual em Saúde-BVS: 1. mobile health, 2. telemedicine, 3. mobile applications, 4. child development, 5. screening, 6. developmental disabilities, 7. surveillance, 8. desenvolvimento infantil, 9. deficiências do desenvolvimento, 10 . rastreio, 11 . vigilância, 12. telemedicina e 13. developmental, combinados seguindo o logarítimo: [(1) OR (2) AND (4] + [(1) OR (2) AND (8) $]+[(5)$ AND (1) OR (2) AND (4) $]+[(10)$ AND (1) OR (12) AND (8)] + [(v) OR (7) AND (4)] $+[(10)$ OR (11) AND (8)] + [(5) AND (6) AND (4) AND (2) $]+[(10)$ AND (4) AND (8) AND (12)] + [(7) AND (4) AND (2) OR (1)] + [(11) AND (8) AND (12) OR (1) $]+[$ (13) AND (5) + (3)] + [(13) AND (7) AND (1)]

A pesquisa gerou o número de 33 publicações que foram refinadas usando o título do artigo e resumo e texto completo. Mediante critério de inclusão e exclusão, artigos cujo foco seja a utilização de instrumentos de vigilância ou rastreio para crianças acima de 5 anos; sem associações diretas à temática proposta; artigos de revisão, anteriores ao ano 2000, e artigos que contemplem instrumento de avaliação do desenvolvimento infantil ou relacionados à transtornos específicos, como autismo, déficit de atenção foram excluídos.

Apenas artigos completos, publicados em inglês e português, literatura cinzenta que adotem instrumento de vigilância e/ou rastreio destinado à faixa etária de zero até 5 anos, em que o instrumento contemple pelos menos um (1) dos seguintes domínios (motor grosso, motor fino, resolução de problemas, linguagem receptiva, linguagem expressiva e socioemocional, pessoal-social e socioemocional) publicados a partir de 2000 em formato de aplicativo móvel foram incluídos.

Após revisão mais detalhada, foram selecionados cinco artigos para a presente revisão entre o período de junho e agosto de 2019. Filtros utilizados em todas as bases de dados: artigos completos, publicados entre as datas de 01 de janeiro do ano 2000 até agosto de 2019. Pesquisas com humanos, crianças, crianças pré-escolares, lactentes, nos idiomas português e inglês, coleção de dados nacionais e internacionais. Os estudos foram listados cronologicamente na tabela a seguir. 


\section{Resultados}

Parker et al. (2012) descrevem a adaptação de um programa de monitoramento da saúde materno-infantil intitulado Text4baby (T4B) para Rússia. Lançado em fevereiro de 2010, é o primeiro serviço de mensagens de texto ( $m$-health) gratuito dos Estados Unidos. O T4B enfoca uma variedade de tópicos críticos de saúde, incluindo imunização, nutrição, saúde mental, saúde bucal, marcos do desenvolvimento e sono seguro. No procedimento de inscrição, é enviado uma mensagem de texto com a data de nascimento esperada para o parto da criança. A partir do cadastro, recebe-se três mensagens por semana com informações baseadas em evidências relevantes para cada estágio da gestação.

Tabela 1

Descrição dos Resultados

\begin{tabular}{|c|c|c|c|c|c|}
\hline Estudo & Objetivo & Participantes & Instrumento & Contexto & Conclusão \\
\hline $\begin{array}{l}\text { Parker et } \\
\text { al., } 2012\end{array}$ & $\begin{array}{l}\text { Descrever proposta } \\
\text { de adaptação da } \\
\text { tecnologia de envio } \\
\text { por mensagem para } \\
\text { monitoramento de } \\
\text { cuidados materno } \\
\text { infantil, Text4Baby na } \\
\text { Rússia. }\end{array}$ & $\begin{array}{c}\text { Não há } \\
\text { participantes }\end{array}$ & $\begin{array}{l}\text { Text4Baby } \\
\text { Vigilância } \\
\text { (marcos do } \\
\text { desenvolvi- } \\
\text { mento infantil). }\end{array}$ & Saúde & $\begin{array}{l}\text { Reforça-se a importância da } \\
\text { necessidade em se conduzir o } \\
\text { acompanhamento epidemiológico } \\
\text { na população materno-infantil e es- } \\
\text { forços na colaboração entre países, } \\
\text { visando orientar a compreensão } \\
\text { internacional no uso das melhores } \\
\text { práticas com tecnologia emergente. }\end{array}$ \\
\hline $\begin{array}{l}\text { Suh et } \\
\text { al., } 2017\end{array}$ & $\begin{array}{l}\text { Descrever o processo } \\
\text { de construção de } \\
\text { um instrumento de } \\
\text { rastreio acessível } \\
\text { por tecnologia móvel } \\
\text { e resultados do } \\
\text { estudo de viabilidade } \\
\text { com a população- } \\
\text { alvo utilizando o } \\
\text { instrumento. }\end{array}$ & $\begin{array}{c}14 \text { famílias } \\
\text { latinas }\end{array}$ & $\begin{array}{l}\text { Baby Steps Text } \\
\text { Rastreio } \\
\text { (pessoal-social, } \\
\text { resolução de } \\
\text { problemas, } \\
\text { motor fino, } \\
\text { motor amplo e } \\
\text { comunicação). }\end{array}$ & Saúde & $\begin{array}{l}\text { Mensagens de texto são ferrametas } \\
\text { viáveis para dar suporte aos pais no } \\
\text { monitoramento e rastreio do desen- } \\
\text { volvimento infantil. A utilização do } \\
\text { sistema de forma continuada para } \\
\text { além do intervalo do estudo indica } \\
\text { uma abordagem promissora no } \\
\text { incentivo e envolvimento dos pais } \\
\text { e cuidadores no acompanhamento } \\
\text { de suas crianças. }\end{array}$ \\
\hline $\begin{array}{l}\text { Maleka } \\
\text { et al., } \\
2016\end{array}$ & $\begin{array}{l}\text { Avaliar a versão } \\
\text { m-health do } \\
\text { instrumento de } \\
\text { triagem Parents } \\
\text { Evaluation } \\
\text { Developmental Status } \\
\text { (PEDS) e PEDS: } \\
\text { Developmen tal } \\
\text { Milestones (PEDS:DM) } \\
\text { para uso de agentes } \\
\text { comunitários de } \\
\text { saúde. }\end{array}$ & $\begin{array}{c}207 \\
\text { participantes }\end{array}$ & $\begin{array}{l}\text { PEDS; PEDS:DM } \\
\text { Rastreio } \\
\text { (Linguagem } \\
\text { expressiva } \\
\text { e receptiva, } \\
\text { motor fino, } \\
\text { motor amplo, } \\
\text { comporta } \\
\text { mento, } \\
\text { autonomia e } \\
\text { aprendizagem. }\end{array}$ & Saúde & $\begin{array}{l}\text { Os resultados indicaram um alto } \\
\text { indice de correspondência positiva } \\
(100 \%) \text { e correspondência nega- } \\
\text { tiva ( } 96 \% \text { ) entre as duas versões } \\
\text { com índice de concordância entre } \\
\text { avaliadores entre as duas condi- } \\
\text { ções do coeficiente Kappa ( } k=0,873 \\
\text { a } k=0,961) \text { sugerem um desfecho } \\
\text { positivo na aplicação entre as } \\
\text { duas versões, habilitando agentes } \\
\text { comunitários de saúde treinados a } \\
\text { aplicar o instrumento. }\end{array}$ \\
\hline $\begin{array}{l}\text { Cheng } \\
\text { et al., } \\
2017\end{array}$ & $\begin{array}{l}\text { Construir e validar } \\
\text { um sistema de } \\
\text { rastreio eletrônico } \\
\text { para detecção } \\
\text { de atrasos no } \\
\text { desenvolvimento } \\
\text { infantil. }\end{array}$ & $\begin{array}{c}120 \\
\text { cuidadores }\end{array}$ & $\begin{array}{l}\text { Taipei II } \\
\text { Rastreio } \\
\text { (marcos do } \\
\text { desenvolvi- } \\
\text { mento) }\end{array}$ & Saúde & $\begin{array}{l}\text { Resultados da avaliação do sistema } \\
\text { revelou excelente concordância } \\
\text { entre a versão multimídia do Taipei } \\
\text { II e a baseada em texto. Os resul- } \\
\text { tados demonstraram que um total } \\
\text { de } 118 \text { (98\%) participantes preferiu } \\
\text { a versão multimídia ou não tinha } \\
\text { preferência, e apenas dois ( } 2 \%) \text { pre- } \\
\text { feriram o versão em papel. No total, } \\
\text { a maioria dos participantes ( } n=114) \\
\text { preferiu plataforma multimídia, } \\
\text { apoiando fortemente sua aceitação. }\end{array}$ \\
\hline
\end{tabular}


Tabela 1 (continuação) Descrição dos Resultados

\begin{tabular}{|c|c|c|c|c|c|}
\hline Estudo & Objetivo & Participantes & Instrumento & Contexto & Conclusão \\
\hline $\begin{array}{l}\text { Warrell } \\
\& \\
\text { Arriaga, } \\
2017\end{array}$ & $\begin{array}{l}\text { Desenvolver o } \\
\text { ActEarly App e reali- } \\
\text { zar estudo de usabili- } \\
\text { dade do aplicativo. }\end{array}$ & $\begin{array}{c}\text { Estudo 1: } 9 \\
\text { participantes } \\
\text { Estudo 2: } 8 \\
\text { participantes } \\
\text { (2 cuidadores) }\end{array}$ & $\begin{array}{l}\text { ActEarly App } \\
\text { (comunica- } \\
\text { ção, cognição, } \\
\text { motricidade, } \\
\text { socioemocio- } \\
\text { nal) }\end{array}$ & Universidade & $\begin{array}{l}\text { Dois estudos de usabilidade foram } \\
\text { conduzidos. } \\
\text { Estudo 1: design e desenvolvimento } \\
\text { de uma solução de aplicativo móvel } \\
\text { como uma ferramenta pública } \\
\text { para acompanhamento de mar- } \\
\text { cos de desenvolvimento; } 2 \text {. design } \\
\text { visual e componentes de fluxo } \\
\text { de trabalho; 3. implicações para } \\
\text { projetar outros instrumentos para } \\
\text { acompanhamento de marcos do } \\
\text { desenvolvimento. } \\
\text { Estudo 2: Resultado positivo do } \\
\text { redesign do estudo 1. Aplicativo } \\
\text { construido para versão Android. }\end{array}$ \\
\hline
\end{tabular}

O lançamento do projeto T4B Rússia foi estimado para dezembro de 2011, em Moscou e São Petersburgo. Por meio do conhecimento prévio sobre sobre envio de mensagens T4B realizados nos EUA, a Rússia também adorá um monitoramento abrangente e pesquisadores da Universidade de Moscou projetarão a análise quantitativa do recibo da mensagem e outras adaptações. Por fim, os autores reforçam a importância da necessidade em se conduzir o acompanhamento epidemiológico na população materno-infantil, além de esforços para colaboração e intercâmbio do uso das melhores práticas de tecnologia emergente entre países.

Também implicado no cenário da tecnologia móvel, o sistema original Baby Steps Text para acompanhamento do progresso do desenvolvimento infantil teve seu formato inicial projetado com software para utilização no Twitter, com trabalhos que datam do período de 2007-2009 (Kientz et al., 2007; Kientz et al., 2009). O Baby Steps Text é um instrumento de rastreio com o propósito de alcançar populações mais carentes, com recursos limitados ou que não tenham acesso a smartphones sofisticados. A ferramenta permite que pais ou cuidadores rastreiem e revisem informações sobre os marcos do desenvolvimento e os conectem a recursos, usando apenas mensagens de texto, expandindo assim o alcance de seus benefícios.

Suh et al. (2017) descreveram os resultados de pesquisas preliminares com a utilização de protótipo e storyboarding do Baby Steps Text, baseado em mensagem de texto (SMS), com quatorze famílias latinas durante um mês de estudo. No artigo, são apresentadas informações do projeto, desenvolvimento e viabilidade do conteúdo no formato SMS. No entanto, não foram examinadas as propriedades psicométricas relacionadas ao instrumento, mas sim da adequação ao formato $m$-health, caracterizando-se como um estudo usabilidade/viabilidade.
As questões inseridas no sistema totalizaram 660 marcos do desenvolvimento. Os itens presentes no questionário foram inspirados no Ages and Stages Questionnaire (ASQ), propondo-se investigar os domínios pessoal-social, comunicação, motor amplo, motor fino e solução de problemas. Quatro opções de respostas foram disponibilizadas: sim, às vezes, ainda não e revisitar. Ao longo do processo de criação e protótipo, três modalidades de acesso foram desenvolvidas em alta fidelidade: mensagem de texto, quiosque público e website.

O estudo para implementação incial do sistema, realizado em um mês, concluiu que as mensagens de texto são ferrametas viáveis para auxiliar os pais no monitoramento e rastreio do desenvolvimento infantil. A utilização do sistema de forma continuada para além do intervalo do estudo indica uma abordagem promissora no incentivo e envolvimento dos pais e cuidadores no acompanhamento de suas crianças.

Para estudos futuros, são encorajados o desenvolvimento de instrumentos de rastreio com versões reduzidas para melhor adaptação dos caracteres para a tecnologia de SMS, tendo em vista que o sistema absorvia 160 caracteres, e a redução dos caracteres originais alteraria a validade do instrumento. $\mathrm{O}$ estudo lança a perspectiva da inclusão de relatório de progresso resumido, envio de atividades para aumentar o engajamento dos pais no acompanhamento da criança, diário de registro fotos dos momentos afetivos da criança.

Ao considerar no contexto alta prevalência de atrasos no desenvolvimento e acesso limitado às avaliações do desenvolvimento em países de baixa renda, Maleka et al. (2016) avaliaram a versão $m$-health do instrumento de triagem Parents Evaluation Developmental Status (PEDS) and PEDS: Developmental Milestones (PEDS:DM) para uso de agentes comunitários de saúde. 
O instrumento PEDS e PEDS: DM são uma ferramenta de triagem por relato dos pais, composto por 10 questões utilizadas para coletar informações sobre o desenvolvimento. O PEDS: DM oferece indicativos de habilidades das crianças nos seguintes domínios do desenvolvimento: linguagem expressiva, linguagem receptiva, motor fino, motor grosso, social-emocional, autonomia e acadêmico. Para que a versão móvel fosse avaliada, foi realizada a correspondência em relação ao método de aplicação.

Foram criados dois grupos de administração do instrumento, um grupo de fonoaudiólogos utilizou a versão convencional "lápis e papel" e outro que foi composto por agentes de saúde comunitários utilizou a versão m-health. Também foi avaliada a confiabilidade entre os avaliadores agentes de saúde comunitários em relação aos fonoaudiólogos.

Com o objetivo de verificar a correspondência entre os dois métodos de aplicação, os agentes de saúde foram inicialmente treinados para utilizar o instrumento na versão desenvolvida para smartphone e posterior administração com 207 cuidadores que frequentavam um espaço para bebês. Em paralelo, um fonoaudiólogo administrou o instrumento "papel e lápis" nos mesmos participantes.

Os resultados indicaram um alto indice de correspondência positiva $(100 \%)$ e correspondência negativa (96\%) entre as duas versões com índice de concordância, os avaliadores e as duas condições do coeficiente kappa ( $k=0,873$ a $k=0,961)$, o que sugeriu um desfecho positivo na aplicação entre as duas versões, habilitando agentes comunitários de saúde treinados a aplicar o instrumento. Vale destacar que a precisão da versão do instrumento no formato para smartphone indica estudos futuros.

Cheng et al. (2017) apresentam o processo de construção de um sistema de rastreio eletrônico para detecção de atrasos no desenvolvimento infantil e validade pós-desenvolvimento do sistema. A construção foi baseada no instrumento para detecção precoce de atraso no desenvolvimento Taipei II (Taipei Preschooler Developmental Checklist 2nd version), utilizado por muitos anos em Taiwan.

Para validar o sistema após o desenvolvimento, esse estudo conduziu um experimento e aplicação de questionário para usuários de pesquisa. Participaram do experimento cinco especialistas e 120 cuidadores de crianças de $6,12,18,30$. A etapa de construção e avaliação de sistema foi orientada por especialistas na área. A etapa de validação clínica de 13 grupos etários de 4 meses a 6 anos foi iniciada em estudos anteriores. Os resultados indicaram que o uso dos checklists baseado em recursos multimídia mantêm com sucesso as propriedades psicométricas do instrumento original baseada em "papel e lápis". O sistema de triagem eletrônica desenvolvido é composto por quatro partes, incluido a interface do site, módulo de triagem eletrônica, banco de dados e módulo gerenciador e funcionalidade de plataforma cruzada.
Após o experimento, os resultados da avaliação do sistema revelou excelente concordância entre a versão multimídia do Taipei II e a baseada em texto. Os resultados demonstraram que um total de 118 (98\%) participantes preferiu a multimídia versão ou não tinha preferência, e apenas $2(2 \%)$ preferiram o versão em papel. No total, a maioria dos participantes $(n=114)$ preferiu plataforma multimídia, apoiando fortemente sua aceitação.

Verificou-se nesse estudo vantagens na utilização de um sistema interativo e animação mostrando-se capazes de oferecer efeitos positivos na aprendizagem na pesquisa em medicina. Os feedbacks dos participantes indicam que o sistema $e$-screening foi bem aceito e mais facilmente acessível que o original formato "papel e lápis". Comparativamente, a triagem regular baseada em texto apresenta alguns aspectos limitantes, como excluir aqueles com baixa alfabetização e aqueles cuja língua nativa é diferente do texto, além de engajar menos a atenção do usuário. Após o experimento, verificou-se que os participantes relataram que o uso do sistema de triagem eletrônica para atraso do desenvolvimento aumentou sua compreensão e manteve-os interessados no procedimento. Embora tenha sido um estudo com resultados de boa aceitação pelos usuários, a amostra foi de 120 participantes, o que sugere estudos futuros. Os autores sugerem para estudos futuros aprimorar as figuras de animação introduzindo algumas caracteristicas para grupos étnicos específicos de Taiwan.

Dando continuidade ao estudos de investigação de Muñoz e Arriaga (2015) sobre as percepções dos pais sobre o uso da tecnologia como uma ferramenta para acessar informações sobre saúde e estudo do conhecimento dos pais sobre acompanhamento dos marcos do desenvolvimento, Warrell e Arriaga (2017) desenvolveram estudos de criação do aplicativo do do programa de saúde pública existente desde 2002, Learn the Signs. Act Early e testes de usabilidade para aplicativo.

Foram realizados dois estudos. No estudo 1, foram oferecidas orientações informativas a nove participantes, todos usam aplicativos móveis com frequência. Nele avaliou-se problemas pontuais de usabilidade e pontos problemáticos do aplicativo Act Early. Os resultados do Estudo 1 geraram outra interface de informação para o Estudo 2. No Estudo 2, as orientações com comandos informativos são executadas por sete participantes, sendo que todos usam aplicativos móveis com frequência. Os questionários de pré-teste e pós-teste foram administrados antes e depois de cada aplicação dos estudos. Os resultados do Estudo 1 revelaram alguns obstáculos ao criar, projetar, avaliar e desenvolver soluções móveis para finalidade de rastreamento de marcos do desenvolvimento uma vez que não havia esse sistema de domínio público disponível. Essa pesquisa tem três principais contribuições: 1. o design e desenvolvimento de uma solução de aplicativo móvel como uma ferramenta pública para acompanhamento de marcos de desenvolvimento, 
2. os resultados dos estudos de usabilidade informando design visual e componentes de fluxo de trabalho e 3. implicações para projetar outros instrumentos para acompanhamento de marcos do desenvolvimento.

O Estudo 2 apresentou resultados positivos em relação ao redesign inicial, com versão do sistema Android disponível e versão futura para Iphone. Embora nesse estudo o grupo de participantes incluidos fossem usuários frequentes e experientes de aplicativos, o acesso aos cuidadores de crianças era limitado, apenas dois participaram. Pesquisas futuras sugerem estudo longitudinal com cuidadores usando mais atualizações e redesign durante esse período.

Esta revisão sistemática teve como objetivo investigar quais foram os artigos que inseriram na metodologia a implementação de instrumento de vigilância ou rastreio para atraso de desenvolvimento por meio de $m$-health. A utilização de aplicativos móveis na área da saúde apresenta benefícios já reconhecidos relacionados à gestão de doenças e à adesão ao tratamento, à ampliação do canal de comunicação entre paciente e agente de saúde, à detecção e ao rastreio de doenças (Ciani et al., 2019; Kientz et al., 2009; Suh et al., 2017). Inovações também estão presentes na ascendência da interseção entre tecnologia e psicologia da saúde e avaliação psicológica (Borrelli e Ritterband, 2015; CFP, 2018).

No entanto, a utilização de instrumentos de rastreio e vigilância do desenvolvimento infantil em formato de dispositivo móvel ( $m$-health) não é uma prática amplamente difundida, o que pode ser aferido pelo baixo número de artigos encontrados na literatura científica sobre estudos com essa modalidade de administração de instrumento destinado à faixa etária até cinco anos. Confirmando ser uma área ainda em expansão e com potenciais desdobramentos (Primi, 2010; Koich Miguel, 2017; Borrelli e Ritterband, 2015).

Ainda assim, pudemos encontrar em nossa pesquisa pelo menos três categorias de estudo: 1. descritivo, 2. usabilidade/viabilidade e 3. validade. Destaca-se que, embora as datas das publicações dos artigos sejam relativamente recentes, a origem dos estudos que permitiram a criação da interface monitoramento e rastreio para atraso do desenvolvimento infantil e tecnologia móvel datam desde 2002, sendo ao longo dos anos realizadas adaptações aos avanços da tecnologia.

$\mathrm{Na}$ categoria de estudo descritivo (Parker et al., 2012), ao apresentar a plataforma interativa Tex4babyRússia como possibilidade do uso da tecnologia móvel na estratégia de monitoramento da saúde materno-infantil e utilização desse recurso para políticas públicas reforça a importância do intercâmbio da tecnologia entre países e participação da iniciativa pública e privada para manutenção desses programas. No entanto, não são inseridas no escopo do artigo informações quanto à generalização desses benefícios e sua eficácia.
Quanto à categoria usabilidade/viabilidade, a pesquisa de Warrell e Arriaga (2017) foi de extrema relevância e pioneirismo ao aproximar tecnologia da informação, interação usuário/tecnologia à solução de necessidades em saúde pública aberta ao domínio público. Embora o estudo de desenvolvimento do aplicativo e usabilidade tenha obtido desfecho positivo na transposição do material de monitoramento do Act Early para versão app, a eficácia da versão em aplicativo do programa de monitoramento Learn the Signs. Act Early no engajamento dos pais para o monitoramento do desenvolvimento infantil não foi explorada, tal como verificada na utilização da versão impressa (Gadomski et al. 2018)

Quanto ao estudo de usabilidade/viabilidade do instrumento de rastreio Baby Steps Text (Suh et al., 2017), apresenta-se como único instrumento de rastreio desenvolvido especificamente para o layout de acesso em formato $m$-health. Oferece grande contribuição na interlocução entre avaliação psicológica e tecnologia da informação, uma vez que se alerta quanto a possíveis obstáculos referentes aos limites da tecnologia na interface com instrumentos de medida, considerando-se que as propriedades psicométricas podem ser afetadas na adaptação de diferentes formatos.

Já os instrumentos Taipei II, PEDS e PEDS-DM com propriedades psicométricas validadas em estudos anteriores em versão "papel e lápis", foram os únicos que realizaram pesquisa de validade correspondente do instrumento com a versão $m$-health. No entanto, mais estudos de precisão dessa versão são necessários.

Ainda que a literatura levantada ao longo da revisão, mas não selecionada para análise diante dos critérios de inclusão e exclusão, identifique uma vasta quantidade de instrumentos para rastreio global do desenvolvimento e domínios específicos e já disponibilizem recursos eletrônicos para instrução, aplicação, correção, acesso remoto ou informatizado, como ASQ, ASQ-SE, Dessa-Mini, SWYC, Battelle Developmental Inventory, Vineland Adaptative Behavior Scales, Denver II, VB-MAPP, Cognoa, as pesquisas envolvendo o Text4 Baby, Baby Steps Text, Taipei II, PEDS e PEDS-DM, CDC Milestones App foram as únicas que atenderam aos critérios de elegibilidade do estudo.

Esta revisão tem algumas limitações que devem ser mencionadas. A busca de artigos a partir dos restritos descritores selecionados e a possível versão para tecnologia móvel de instrumentos de vigilância e rastreio sem a publicação em periódicos científicos pode ter influenciado os resultados. Conclui-se que mais estudos sejam necessários, especialmente para avaliar o impacto e a eficácia do uso de instrumentos de monitoramento e rastreio para atraso no desenvolvimento infantil em formato de tecnologia móvel, especialmente com populações de culturas distintas e países em desenvolvimento.

Visto a ampliação do potencial de acesso à informação por meios de dispositivos móveis no Brasil (CGI, 2018), associado às evidências levantadas nestes estudos 
sugerirem que a interface desses instrumentos a dispositivos móveis pode ser utilizada como mecanismo auxiliar para pais, cuidadores e agentes de saúde no monitoramento, rastreio e identificação precoce do atraso no desenvolvimento, estima-se que este levantamento possa contribuir no desenvolvimento de futuras pesquisas de construção de instrumentos, utilizando tecnologia móvel para a realidade brasileira.

Finalmente, reforça-se que, embora o ritmo guiado pela tecnologia seja acelerado, o modelo de criação de instrumentos eletrônicos de monitoramento e rastreio deve seguir o padrão criterioso de desenvolvimento de instrumentos psicológicos, para que assim sejam considerados válidos e confiáveis ao medir ou inferir o que se pretende investigar, isto é, assegurar as propriedades psicométricas na administração nesses formatos (CFP, 2018, 2019). Portanto, é de extrema importância que o intercâmbio entre tecnologia da informação e avaliação psicológica possam caminhar juntas para a consolidação de novas propostas, a fim de aprimorar o alcance de ferramentas de avaliação, rastreio e monitoramento.

\section{Agradecimentos}

Não há menções.

\section{Financiamento}

Todas as fontes de financiamento para elaboração e produção do estudo (coleta, análise e interpretação dos dados, bem como, escrita dos resultados no presente no manuscrito) foram fornecidas pelo projeto de pesquisa "Capes cod.001CNPq e FAPERJ".

\section{Contribuições dos autores}

Todos os autores participaram da elaboração do manuscrito. Especificamente, o(s) autor(es) Flávia Monteiro Pereira, Helenice Charchat e J. Landeira participou(ram) da redação inicial do estudo - conceitualização, investigação, visualização, o(s) autor(es) Flávia Monteiro Pereira, Helenice Charchat e J. Landeira participou(ram) da análise dos dados, e o(s) autor(es) Flávia Monteiro Pereira, Helenice Charchat e J. Landeira participou(ram) da redação final do trabalho - revisão e edição.

\section{Disponibilidade dos dados e materiais}

Todos os dados e sintaxes gerados e analisados durante esta pesquisa serão tratados com total sigilo devido às exigências do Comitê de Ética em Pesquisa em Seres Humanos. Porém, o conjunto de dados e sintaxes que apoiam as conclusões deste artigo estão disponíveis mediante razoável solicitação ao autor principal do estudo.

\section{Conflito de interesses}

Os autores declaram que não há conflitos de interesses.

\section{Referências}

Agency for Health Care Research and Quality (2018). Introducing electronic screening tools for developmental delay and autism into pediatric primary care. Rockville, MD.

Becker, D., Van Breda, W., Funk, B., Hoogendoorn, M., Ruwaard, J., \& Riper, H. (2018). Predictive modeling in e-mental health: A common language framework. Internet Interventions, 12, 57-67. https://doi.org/10.1016/j.invent.2018.03.002

Borrelli, B., \& Ritterband, L. M. (2015). Special issue on eHealth and mHealth: Challenges and future directions for assessment, treatment, and dissemination. Health Psychology, 34, 1205-1208. https://doi.org/10.1037/hea0000323

CDC, Development, N., \& Development, A. (2017). CDC: “Learn the Signs, Act Early." Notes, 35-36.

Centro Regional de Estudos para o Desenvolvimento da Sociedade da Informação, Cetic, (2017). Pesquisa sobre o Uso das Tecnologias de Informação e Comunicação nos Domicílios Brasileiros - TIC Domicílios 2017.

Charchat-Fichman, H., Uehara, E., \& Santos, C. F. (2014). New Technologies in Assessment and Neuropsychological Rehabilitation. Temas Em Psicologia, 22(3), 539-553. https://doi.org/10.9788/TP2014.3-01

Cheng, H. Y. K., Chang, H. T., Huang, P. H., Ju, Y.Y., Chen, L. Y., \& Tseng, K. C. (2017). The Design and Validation of a Child Developmental e-Screening System. Journal of Medical Systems, 41(67), 1-11. https://doi.org/10.1007/s10916-017-0701-z

Ciani, O., Cucciniello, M., Petracca, F., Apolone, G., Merlini, G., Novello, S., Pedrazzoli, P., Zilembo N., Broglia, C., Capalletto, E., Garassino, M., Nicod, E., \& Tarricone, R. (2019). Lung Cancer App (LuCApp) study protocol: A randomised controlled trial to evaluate a mobile supportive care app for patients with metastatic lung cancer. BMJ Open, 9(2), e025483, 1-10. https://doi.org/10.1136/ bmjopen-2018-025483

Comitê Gestor da Internet no Brasil. (2018). ICT Households 2017 - Survey on the use of information and communication technologies in brazilian households

Conselho Federal de Psicologia (2018). Resolução CFP n¹1/2018 - Regulamenta a prestação de serviços psicológicos realizados por meios de tecnologias da informação e da comunicação e revoga a Resolução CFP no 11/2012. CFP. https://e-psi.cfp.org.br/resolucao-cfp-no-11-2018/

Conselho Federal de Psicologia. (2019). Nota técnica n 7/2019/GTEC/CG. CFP. https://site.cfp.org.br/wp-content/uploads/2019/10/NotaT\%C3\%A9cnica-CFP-07.2019.pdf

Duby, J. C., Lipkin, P. H., Macias, M. M., Wegner, L. M., Duncan, P., Hagan, J. F., Cooley, W. C., Swigonski, N., Biondich, P. G., Lollar, D., Ackermann, J., Brin, A., Crane, M., Gibson, A., Skipper, S. M., Steinberg-Hastings, D., \& Capers, M. (2006). Identifying Infants and Young Children with Developmental Disorders in the Medical Home: An Algorithm for Developmental Surveillance and Screening. Pediatrics, 118(1), 405-420. https://doi.org/10.1542/peds.2006-1231

Durkin, M. (2002). The epidemiology of developmental disabilities in low-income countries. Mental Retardation and Developmental Disabilities Research Reviews, 8(3), 206-211. https://doi.org/ 10.1002/mrdd.10039

Gadomski, A. M., Riley, M. R., Scribani, M., \& Tallman, N. (2018). Impact of "learn the Signs. Act Early." Materials on parental engagement and doctor interaction regarding child development. Journal of Developmental and Behavioral Pediatrics, 39(9), 693-700. https://doi. org/10.1097/DBP.0000000000000604 
Grantham-McGregor, S., Cheung, Y. B., Cueto, S., Glewwe, P., Richter, L., \& Strupp, B. (2007). Developmental potential in the first 5 years for children in developing countries. Lancet, 369(9555), 60-70. https://doi.org/10.1016/S0140-6736(07)60032-4

Hagan J. F., Shaw J. S., \& Duncan P. M. (2017). Bright futures: Guidelines for health supervision of infants, children, and adolescents (4th ed). American Academy of Pediatrics.

Halle, T., Zaslow, M., Wessel, J., Moodie, S., \& Darling-Churchill, K. (2011). Understanding and Choosing Assessments and Developmental Screeners for Young Children Ages 3-5: Profiles of Selected Measures. Office of Planning, Research, and Evaluation, Administration for Children and Families, U.S. Department of Health and Human Services https://files.eric.ed.gov/fulltext/ED566870.pdf

International Test Commission (2005). International Guidelines on Computer-Based and Internet Delivered Testing. https://www. intestcom.org/files/guideline_computer_based_testing.pdf

Johnson, C. P., \& Blasco, P. A. (19977). Infant Growth and Development. Pediatrics in Review, 18(7), 224-242. https://doi.org/10.1542/pir.18$7-224$

Kientz, J. A., Arriaga, R. I., \& Abowd, G. D. (2009). Baby steps: Evaluation of a system to support record- keeping for parents of young children. Proceedings of the SIGCHI Conference on Human Factors in Computing Systems. https://doi.org/10.1145/1518701.1518965

Kientz, J. A., Arriaga, R. I., Chetty, M., Hayes, G. R., Richardson, J., Patel, S. N., \& Abowd, G. D. (2007). Grow and know: Understanding record-keeping needs for tracking the development of young children. Proceedings of the SIGCHI conference on Human factors in computing systems. https://doi.org/10.1145/1240624.1240830

Koich Miguel in Damásio, B. F., \& Borsa, J. C. (2017). Manual de desenvolvimento de instrumentos psicologicos. Vetor Editora,1. ed.

Maleka, B. K., Van Der Linde, J., Glascoe, F. P., \& Swanepoel, D. W. (2016). Developmental Screening-Evaluation of an m-Health Version of the Parents Evaluation Developmental Status Tools. Telemedicine and E-Health, 22(12), 1013-1018. https://doi.org/10.1089/ tmj.2016.0007

McLean, K., Goldfeld, S., Molloy, C., Wake, M., \& Oberklaid, F. (2014). Screening and suerveillance in early childhood health: Rapid review of evidence for effectiveness and efficiency of models. Murdoch Childrens Research Institute. https://www.health.nsw.gov.au/kidsfamilies/ MCFhealth/Documents/screening-and-surveillance-in-early-childhood.pdf

Moher, D., Liberati, A., Tetzlaff, J., \& Altman, D. G. (2009). Preferred reporting items for systematic reviews and meta-analyses: The PRISMA statement. Journal of Clinical Epidemiology, 62(10), 1006-1012. https://doi.org/10.1016/j.jclinepi.2009.06.005

Moodie, S., Daneri, P., Goldhagen, S., Halle, T., Green, K., \& LaMonte, L. (2014). Birth to 5: Watch Me Thrive! A Compendium of Screening Measures for Young Children. Office of Planning, Research and Evaluation, Administration for Children and Families, U.S. Department of Health and Human Services. https://www.acf.hhs.gov/sites/default/files/documents/ecd/screening_compendium_march2014.pdf

Muñoz, D., \& Arriaga, R. I. (2015). Low Income Parent's Values Involving the Use of Technology. Em J. Abascal, S. Barbosa, M. Fetter., T. Gross, P. Palanque, \& M. Winckler (Eds.), Human-computer interaction - INTERACT 2015: 15th IFIP TC 13 International Conference Bamberg, Germany, September 14-18, 2015 proceedings, Part III (pp. 53-70). Springer.

Organização Pan-Americana da Saúde (2005). Organização Pan-Americana da Saúde Manual para vigilância do desenvolvimento infantil no contexto da AIDPI. Washington, D.C.

Parker, R. M., Dmitrieva, E., Frolov, S., \& Gazmararian, J. A. (2012). Text4baby in the United States and Russia: An opportunity for understanding how mHealth affects maternal and child health. Journal of Health Communication, 17, 30-36. https://doi.org/10.1080/10 810730.2011.649162

Petersen, M. C., Kube, D. A., \& Palmer, F. B (1998). Classification of developmental delays. Seminars in Pediatric Neurology, 5(1), 2-14. https:// doi.org/10.1016/S1071-9091(98)80012-0

Pizur-Barnekow, K., Erickson, S., Johnston, M., \& Bleuel, D. (2010). Early Identification of Developmental Delays Through. Infants E Young Children, 23(4), 323-330. https://doi.org/10.1097/IYC.0b013e3181f422a4

Primi, R. (2010). Avaliação psicológica no Brasil: Fundamentos, situação atual e direções para o futuro. Psicologia: Teoria e Pesquisa, 26(spe), 25-3. https://doi.org/10.1590/S0102-37722010000500003

Shevell M., Ashwal S., Donley D., Flint J., Gingold M., \& Hirtz D. (2003). Practice parameter: Evaluation of the child with global developmental delay. Report of the Quality Standards Subcommittee of the American Academy of Neurology and The Practice Committee of the Child Neurology Society Neurology. Neurology, 60(3), 367-80. https://doi.org/10.1212/01.WNL.0000031431.81555.16

Shonkoff, J. P., \& Meisels, S. J. (2000). Handbook of early childhood intervention (2nd ed). Cambridge University Press.

Suh, H., Porter, J. R., Racadio, R., Sung, Y. C., \& Kientz, J. A. (2017). Baby Steps Text: Feasibility Study of an SMS-Based Tool for Tracking Children's Developmental Progress. AMIA ... Annual Symposium Proceedings. AMIA Symposium, 2016, 1997-2006.

Twomey, D. M., Wrigley, C., Ahearne, C., Murphy, R., De Haan, M., Marlow, N., \& Murray, D. M. (2018). Feasibility of using touch screen technology for early cognitive assessment in children. Archives of Disease in Childhood, 103(9), 853-858. https://adc.bmj.com/ content $/ 103 / 9 / 853$

Warrell, L., \& Arriaga, R. I. (2017). ActEarly: Evaluation and redesing of a mobile application for tracking developmental milestones. Atlanta, Georgia: School of Interactive Computing Georgia Institute of Technology.

\section{Sobre os autores}

Flávia Monteiro Pereira é psicóloga clínica, mestre e doutoranda em Psicologia Clínica e Neurociências pela PUC-Rio.

Helenice Charchat Fichman é psicóloga graduada pela UFRGS, mestrado e doutorado pelo Instituto de Psicologia da USP. Atualmente, é professora associada do Departamento de Psicologia (graduação e pós-graduação) da PUC-RJ.

J. Landeira-Fernandez é psicólogo pela Pontifícia Universidade Católica do Rio de Janeiro (PUC-Rio). Mestre em Psicologia experimental pela Universidade de São Paulo e PhD pela UCLA. Professor Titular do Departamento de Psicologia da PUC-Rio, onde é o atual diretor. Pesquisador nível 1A pelo Conselho Nacional de Desenvolvimento Científico e Tecnológico (CNPq) e Fundação de Amparo à Pesquisa do Estado do Rio de Janeiro (FAPERJ).

\section{Como citar este artigo}

Pereira, Charchat-Fichman, \& Landeira-Fernandez (2021). Instrumentos de Vigilância e Rastreio do Desenvolvimento Infantil e Tecnologia Móvel: Revisão. Avaliação Psicológica, 20(1), 71-79. http://dx.doi.org/10.15689/ap.2021.2001.18897.08 